\title{
COMPARACIÓN DE LA COMPRENSIÓN LECTORA EN ALUMNOS DE CUARTO AÑO DE SECUNDARIA DE CENTROS EDUCATIVOS ESTATALES Y NO ESTATALES DE LIMA METROPOLITANA
}

\author{
READING COMPREHENSION COMPARISON BETWEEN FOURTH GRADE HIGH SCHOOL \\ STUDENTS OF PUBLIC AND PRIVATE SCHOOLS OF LIMA METROPOLITANA \\ Ana Delgado V. ${ }^{1}$, Luis Escurra M., María Atalaya P., Juan Pequeña C., Abel Cuzcano, \\ Carmen Álvarez T., Rosa Rodríguez T. \\ Universidad Nacional Mayor de San Marcos, Perú
}

(RECiBido el 2/07/2010, ACEPTAdo El 5/10/2010)

\begin{abstract}
RESUMEN
Objetivos: a. Estudiar el desarrollo de la comprensión lectora en alumnos de cuarto año de secundaria. b. Analizar el nivel de desarrollo de la comprensión de los alumnos de centros educativos estatales y no estatales en cuarto de secundaria. c. Comparar el nivel de desarrollo de la comprensión lectora por género en cuarto año de secundaria. d. Establecer las propiedades psicométricas del instrumento utilizado. e. Construir los baremos para los alumnos del cuarto año de secundaria, considerando las variables género y tipo de gestión del centro educativo. f. Estudiar el modelo del constructo de la comprensión lectora utilizando el análisis factorial confirmatorio. Diseño: Estudio descriptivo comparativo, llevado a cabo en alumnos de cuarto año de secundaria considerando centros educativos estatales y no estatales, y considerando el género. Material y Métodos: Muestreo por conglomerados en dos etapas, con probabilidades proporcionales al tamaño; la muestra estuvo constituida por 613 participantes de las siete UGEL de Lima Metropolitana. Se elaboró una Prueba de Comprensión Lectora para el cuarto de secundaria. Se llevó a cabo el estudio psicométrico del instrumento para evaluar la validez y la confiabilidad. Asimismo, se llevó a cabo el estudio descriptivo e inferencial de las variables estudiadas. Resultado: el 64,77\% de los alumnos de cuarto año de secundaria pertenecían a colegios estatales mientras que el 35,23\% provenían de colegios privados. El 46,33\% de los estudiantes de cuarto de secundaria fueron varones mientras que el $53,67 \%$ fueron mujeres. Conclusiones: se ha encontrado que el instrumento creado es válido y confiable. Se encontraron diferencias estadísticamente significativas en el nivel de comprensión lectora entre los alumnos de colegios estatales y no estatales. Se han elaborado baremos diferenciados para cada tipo de colegio.
\end{abstract}

Palabras clave: Comprensión lectora, alumnos de cuarto año de secundaria, centros educativos estatales, centros educativos no estatales, validez, confiabilidad, baremos.

\begin{abstract}
Objectives: a. To study the reading comprehension development on fourth grade high school students. b. To analyze the comprehension developmental level of fourth grade high school
\end{abstract}

1 Profesora Principal de la Facultad de Psicología de la UNMSM. E-mail: aedvdt2@yahoo.com 
students considering public and private schools. c. To compare the reading comprehension level considering student's gender on fourth grade high school students. d. To study the psychometric properties of the research instrument. e. To study the reading comprehension construct using the confirmatory factorial analysis. Design: Descriptiv.comparative study carried on fourth grade high school students of public and private schools considering students gender. Materials and methods: A b.etapic conglomerate sampling affixed to size, the participants were 613 students of seven units (UGEL) of Lima city. It was constructed a reading comprehension test for fourth level high school students. The statistical analysis considered three stages: Psychometric study in order to calculate validity and reliability index, descriptive indicators, and inferential hypothesis testing. Results: $64,77 \%$ fourth grade high school students were from public schools while $35,23 \%$ were from private schools. $46,33 \%$ fourth grade high school students were male while $53.67 \%$ were female. Conclusions: It has been found that the instrument is valid and reliable. It was found statistically differences on reading comprehension level considering public and private schools (higher on these ones). The fourth grade high school students of private schools have more reading comprehension on literal and inferential reading. However there were no differences between male and female students. Norms have been constructed for each kind of school.

Keywords: Reading comprehension, fourth high school students, public schools, private schools, validity, reliability, norms.

\section{INTRODUCCIÓN}

Uno de los mayores problemas de los estudiantes peruanos es la dificultad para comprender lo que leen, tal como lo demuestra el hecho que el $54 \%$ de nuestros educandos que participaron en la prueba PISA se encontraban en el nivel 0 de la Escala de Alfabetización Lectora de Pisa del 2003, es decir, más de la mitad de los 4,429 participantes peruanos no lograron aprobar las pruebas de nivel 1 , lo que indica que apenas eran capaces de llevar a cabo tareas muy sencillas de localización y jerarquización de datos muy explícitos en textos breves cuya estructura es muy evidente. Según este mismo informe, el $80 \%$ de los participantes se encontraba debajo del nivel 2 , lo que significa que estos estudiantes presentaban mucha dificultad para utilizar la lectura como una herramienta efectiva para ampliar sus conocimientos y habilidades en otras áreas. Esto podría generarles dificultades en la transición del colegio al trabajo, en el aprovechamiento de los estudios superiores y en otras situaciones a lo largo de su vida (Amorós, 2006).

De otro lado, los resultados de la Evaluación Nacional de Comprensión de Textos del 2004 indican que sólo $12,1 \%$ de los estudiantes que culminan el sexto grado de primaria y el $15,1 \%$ de los alumnos que terminan el tercero de secundaria logran los aprendizajes que se esperan para sus respectivos grados escolares, lo cual significa que la mayoría muestra serias dificultades para ubicar datos sencillos en un texto simple y comprender textos explícitos, o reflexionar al menos a un nivel muy básico acerca del contenido.

Diversos autores como Dubois (1991), Cairney (1992), Almeyda y Yataco (1999), Defior, (2000), Gandolfi (2002), Kintsch, Van Dijk, Beck y Carpenter (citados en Echevarría y Gastón, 2004), Vieiro y Gómez (2004), Vallés (2005) y Fons (2006) señalan que leer, no es el simple acto mecánico de descifrar el código de la letra impresa, de agruparlas en sílabas, palabras y frases, sino una construcción activa del sujeto, un acto de razonamiento en el que utiliza claves y estrategias, que le van a permitir construir la 
interpretación del mensaje escrito a partir de la información que le proporciona el texto y los conocimientos que él posee. Leer implica comprender (para lo cual se emplean tanto las relaciones semánticas como las referenciales que se encuentran en el texto), interpretar, descubrir y disfrutar. Para poder comprender lo que se lee es necesario utilizar procesos y estrategias (como planificar, verificar, evaluar, revisar y ensayar), las cuales muchas veces no son enseñadas en la escuela o el alumno aprende a utilizarlas de manera muy limitada. Pero si la lectura se quedara en el nivel comprensivo sería sólo un acto informativo, puntual y estático. Es gracias a la reflexión que la lectura tiene una dimensión dinámica y formativa. Entonces, cuando una persona lee construye de nuevo a partir de los signos gráficos las imágenes, los sentimientos y los pensamientos expresados en el texto, pero los impregna con su subjetividad, es decir, reconstruye el texto de manera significativa. La lectura es entonces una actividad compleja en la cual se van a actualizar conocimientos previos, se hacen anticipaciones, se confronta lo nuevo con lo que ya se ha adquirido, se generan hipótesis y se debe ser capaz de verificarlas o refutarlas, dándose todo esto en un movimiento permanente de avance y retroceso del lector sobre el texto.

La comprensión lectora tiene diversos niveles, en la taxonomía de Barret (Sánchez, 1998) se consideran los siguientes:

El nivel de comprensión literal; es la capacidad de para recordar escenas tal y como son presentadas en el texto. En este nivel el lector debe demostrar su capacidad para repetir las ideas principales, los detalles y las secuencias de los acontecimientos. El nivel de comprensión inferencial, quien lee debe ser capaz de reconstruir el significado de la lectura y relacionarlo con sus propias experiencias, con el conocimiento que tenga del tema y debe plantearse hipótesis o hacer inferencias.

Luego se presenta el nivel de comprensión reorganizativa, consiste en tener la capacidad de sintetizar, esquematizar o resumir la información recibida, siendo capaz de consolidar o reordenar las ideas a partir de la información del texto con la finalidad de hacer una síntesis comprensiva de ella. Finalmente, el nivel de comprensión crítica o evaluativa, se refiere a que el lector pueda deducir, expresar su opinión y emitir juicios a partir de lo leído.

Otro punto de vista es el de Catalá, Catalá, Molina y Monclús (2001) quienes consideran que la lectura comprende las siguientes dimensiones cognitivas: la comprensión literal, la reorganización de la información, la comprensión inferencial y la comprensión crítica o de juicio, e indican que un buen lector hace uso de todas estas dimensiones de manera simultánea conforme lee.

Según Sánchez (2004) y Amorós (2006), la competencia en lectura no sólo se refiere a la decodificación y la comprensión literal, sino que implica la comprensión, el uso y la reflexión de la información escrita para diferentes propósitos, teniendo en cuenta el papel activo e interactivo que, tiene el lector en la adquisición del conocimiento del texto escrito. Para estos autores la verdadera lectura es la de nivel inferencial y crítica, es decir, aquella donde el lector demuestra su capacidad lectora siendo capaz de leer lo que no se encuentra de manera explícita en el texto, reconociendo el lenguaje figurado, comprendiendo el texto de manera global, reconociendo tanto las intenciones del autor 
como la superestructura del texto, pudiendo tomar una postura al respecto, e integrando la nueva información con la que ya posee, haciendo un resumen del texto.

Por lo tanto, la enseñanza de la lectura debe apuntar a llegar al nivel de comprensión crítica, sin embargo, muchas veces se observa que cuando ésta se lleva a cabo se reduce a una actividad en la que los profesores se limitan a hacer preguntas de nivel literal sobre el contenido de un texto, con lo cual los estudiantes no se ven precisados a utilizar su capacidad de inferencia, ni su análisis crítico para comprender un texto (Almeyda y Yataco, 1999).

Fons (2006) señala que la enseñanza y el aprendizaje de la lectura debe darse a lo largo de toda la escolaridad y desde todas las áreas, lamentablemente esto no se cumple en la mayoría de las instituciones educativas. Muchas veces se cree que esto sólo corresponde a los primeros grados de educación primaria y al área de comunicación integral. El sistema escolar está concentrado en lograr que los alumnos descifren el alfabeto y en "obligarlos" a leer, en lugar de que aprendan a leer y hacer que la lectura sea para ellos un hábito que los acompañe siempre, que les resulte gratificante y sea un instrumento que les permita entender mejor la realidad y para adquirir conocimientos útiles en su vida diaria (Sánchez, 2004).

En cuanto a la evaluación de la comprensión lectora, autoras como Catalá et al. (2001) plantean que ésta debe estar plenamente integrada en la actividad escolar, debido a que el aprendizaje de la comprensión lectora supone que el maestro debe ser capaz de activar en los estudiantes sus conocimientos previos, debe llevar a cabo la inducción guiada que les permita construir nuevos conocimientos y les permita jerarquizarlos, consolidarlos y generalizarlos. Por lo tanto, la evaluación debe estar integrada en las diferentes fases y puede llevarse a cabo de manera formal o informal. Una manera de hacerlo es empleando pruebas estandarizadas que perrmiten comprobar si el nivel de comprensión lectora del alumno corresponde al esperado para su grupo normativo (tomando en cuenta el grado escolar, la edad, el género, el tipo de colegio de procedencia, etc). A través de esta evaluación se conoce cuáles son las habilidades que el alumno domina y las que aún no; de acuerdo a estos resultados es posible elaborar el programa recuperativo de las habilidades en las que el estudiante se encuentra en déficit.

Teniendo en cuenta la importancia de la comprensión lectora en el desarrollo académico, ya que es una de las bases del proceso de aprendizaje; considerando los resultados de las evaluaciones internacionales y nacionales, que evidencian un elevado número de estudiantes que presentan dificultades en esta área y habiéndose llevado a cabo estudios de esta variable en los seis grados de educación primaria y en los tres primeros años de secundaria que han permitido contar con instrumentos válidos y confiables para estos grados de estudio y asimismo conocer cómo se dan las diferencias en los niveles de comprensión lectora en éstos grados de estudio entre los alumnos de centros educativos estatales y no estatales, la finalidad de la presente investigación es estudiar el desarrollo de la comprensión lectora en alumnos de cuarto año de secundaria de Lima Metropolitana, considerando el tipo de centro educativo y el género. 


\section{MÉTODO}

En el presente estudio se ha utilizado un diseño de carácter descriptivo comparativo, pues se hace referencia a los alumnos de cuarto año de secundaria de Lima Metropolitana considerando centros educativos estatales y no estatales, y el género de los participantes (Sánchez y Reyes, 2006, Alarcón, 2008).

El universo de investigación estuvo conformado por los alumnos matriculados en cuarto año de secundaria de Lima Metropolitana. Participaron 613 estudiantes. Para la obtención de la muestra se utilizó un procedimiento de muestreo probabilístico b.etápico, en la primera etapa, en cada una de las UGEL se elegió de manera aleatoria los centros educativos a ser incluidos en el estudio, y luego en cada colegio se eligió de manera aleatoria a los participantes del cuarto año de secundaria que fueron evaluados.

Se creó la Prueba de Comprensión Lectora de Delgado, Escurra y Torres (DET) para alumnos de cuarto de secundaria. La cual está constituida por 5 subtests cuyos ítems se encuentran ordenados con nivel de dificultad creciente. El primer subtest está conformado por el texto titulado "Madre Teresa de Calcuta" y consta de 9 ítems, el segundo subtest se denomina "Marie Curie" y consta de 9 ítems, el tercer subtest se denomina "Johannes Gutenberg" y posee 9 ítems, el cuarto subtest se titula "Louis Pasteur" y tiene 9 ítems, y el último subtest denominado "Albert Einstein" posee 8 ítems. En todos los subtests se han considerado ítems que evalúan los niveles de comprensión lectora literal e inferencial. La prueba puede ser administrada de manera individual o colectiva.

Tanto los textos como las instrucciones de los subtests deben ser leídas en silencio por los estudiantes. El examinador se debe limitar a orientar a los alumnos dejándolos en condiciones de trabajar en forma autónoma. Se les debe advertir que pueden volver a leer el texto si fuese necesario.

Los datos fueron procesados utilizando el programa estadístico SPSS. El análisis estadístico se llevó a cabo en tres etapas. En la primera etapa se realizó el análisis psicométrico del instrumento, tomando en cuenta lo propuesto por Marín (1986), así se llevó a cabo el análisis de ítems, se determinó la confiabilidad a través del coeficiente alfa de Cronbach; y la validez de constructo se estableció a través del análisis factorial confirmatorio. En la segunda etapa se realizó un análisis descriptivo de las variables estudiadas, efectuándose el análisis de la normalidad de los puntajes a través de la prueba de Kolmogorov-Smirnov. En la tercera etapa se efectuaron las comparaciones de medias de las variables estudiadas considerando el tipo de colegio y el sexo de los participantes.

\section{RESULTADOS}

\section{Análisis psicométrico}

Se llevó a cabo el análisis de ítems de los cinco subtests que conforman la prueba (ver Tabla 1), encontrándose que todas las correlaciones Íte.test corregidas alcanzan valores que oscilan entre ,44 y ,78, superiores al criterio propuesto por Kline $(1986,1995)$ de ser mayores a ,20, lo cual indica que todos los subtests son consistentes entre sí.

El análisis de la confiabilidad permite señalar que el instrumento es confiable, pues se obtiene un coeficiente alfa de Cronbach de ,77, tal como se presenta en la Tabla 1. 
Tabla N. ${ }^{\circ}$ 1. Análisis de la Confiabilidad de la Prueba de Comprensión Lectora DET para el cuarto año de secundaria.

\begin{tabular}{lcccc}
\hline & $\begin{array}{c}\text { Media de la escala si se } \\
\text { elimina el elemento }\end{array}$ & $\begin{array}{c}\text { Varianza de la escala si } \\
\text { se elimina el elemento }\end{array}$ & $\begin{array}{c}\text { Correlación element. } \\
\text { total corregida }\end{array}$ & $\begin{array}{c}\text { Alfa de Cronbach si } \\
\text { se elimina el elemento }\end{array}$ \\
\hline Subtest 1 & 16,23 & 24,28 &, 44 &, 76 \\
Subtest 2 & 15,30 & 21,69 &, 47 &, 74 \\
Subtest 3 & 14,08 & 20,94 &, 47 &, 74 \\
Subtest 4 & 13,03 & 17,75 &, 75 &, 65 \\
Subtest 5 & 10,37 & 11,10 &, 76 &, 66 \\
\hline \multicolumn{5}{c}{ Alfa de Cronbach $=, 77$} \\
\hline
\end{tabular}

$\mathrm{n}=613$

\section{Análisis de la Validez de Constructo}

La validez de constructo se estudió utilizando el método del análisis factorial confirmatorio aplicando el programa Amos 5.0. De acuerdo a las recomendaciones propuestas por Byrne $(1989,1998)$, se contrastó el modelo teórico propuesto de un factor, con un modelo alternativo que asumía la existencia de valores independientes entre sí, los resultados presentados en la Tabla 2 y el Gráfico 1, indican un valor de chi cuadrado mínimo no significativo $\left(X^{2}=6,83 \mathrm{p}>.05\right)$ y una proporción pequeña $(1,36)$ entre el ch.cuadrado mínimo y los grados de libertad lo cual indica que el modelo propuesto es adecuado.

Asimismo, el índice del ajuste normalizado Delta 1 (NFI Delta 1) fue igual a ,99, el índice comparativo de ajuste (GFI) fue de, 99 y la media de cuadrados del error de aproximación (RMSEA) fue de, 025 , hallazgos que corroboran que el modelo propuesto de 1 factor es válido, con lo cual se puede concluir que la prueba de Comprensión Lectora DET para el cuarto año de secundaria, tiene validez de constructo.

Tabla N. ${ }^{\circ}$ 2. Análisis de la Validez de Constructo de la Prueba de Comprensión Lectora DET para el cuarto año de secundaria a través del Análisis Factorial Confirmatorio.

\begin{tabular}{lcc}
\hline \multicolumn{1}{c}{ Datos } & Modelo de 1 factor & Modelo independiente \\
\hline Parámetros & 10 & 5 \\
Ch.cuadrado mínimo & 6,83 & 542,17 \\
g.l. & 5 & 10 \\
p &, 233 &, 000 \\
Ch.cuadrado mínimo/g.1. & 1,36 & 54,21 \\
NFI Delta1 &, 99 &, 00 \\
RMR &, 04 &, 765 \\
GFI &, 99 &, 66 \\
AGFI &, 98 &, 49 \\
RMSEA &, 025 &, 29 \\
\hline
\end{tabular}

$\mathrm{n}=613$ 


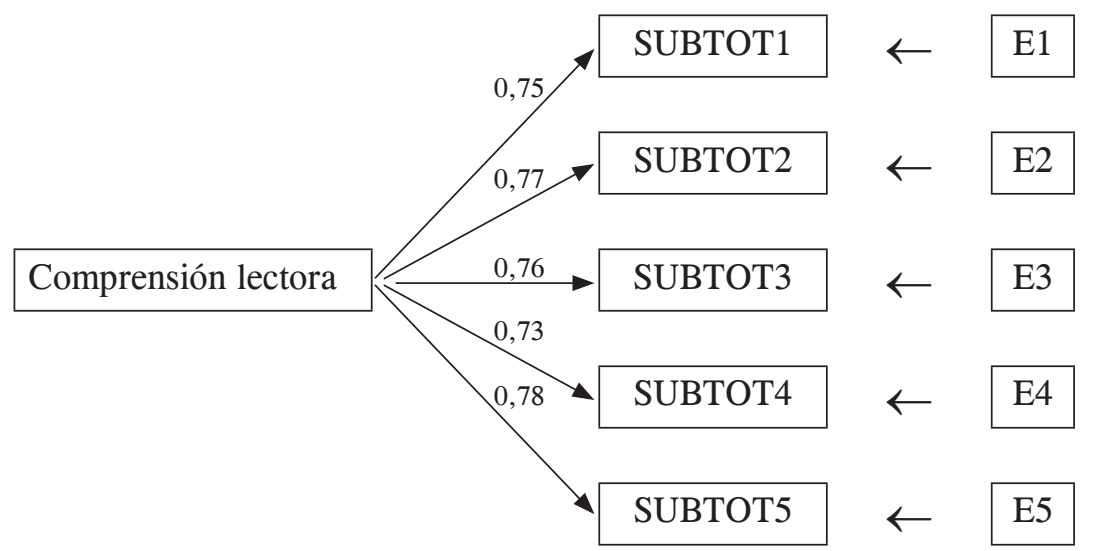

Figura N. ${ }^{\circ}$ 1. Análisis Factorial Confirmatorio de la Prueba de Comprensión Lectora DET para el $4{ }^{\circ}$ Año de Secundaria.

Los resultados obtenidos en el análisis de la confiabilidad y la validez de la Prueba de Comprensión Lectora DET para el cuarto año de secundaria permiten señalar que el instrumento es confiable y válido, con lo cual cumplen con los requerimientos psicométricos básicos para este tipo de instrumentos (Delgado, Escurra y Torres, 2006; Martínez Arias, 1985; Muñiz, 1996 y Anastasi y Urbina, 1998).

\section{Análisis descriptivo}

Los resultados del análisis de la bondad de ajuste a la curva normal fueron realizados a través de la prueba de Kolmogorov-Smirnov (ver Tabla 3), e indicaron valores K-S Z significativos, por lo que se pudo concluir que las distribuciones de los valores analizados no se aproximan a la curva de distribución normal, por cual se utilizaron estadísticos no paramétricos en el análisis de los datos de la prueba de comprensión lectora para el cuarto año de secundaria (Siegel y Castellan, 1995), para una media de 17,25 y desviación estándar (DE) de 5,31.

Tabla N. ${ }^{\circ}$ 3. Análisis de la Bondad de Ajuste a la Curva Normal de Kolmogorov-Smirnov de los Valores Estudiados.

\begin{tabular}{lccc}
\hline \multicolumn{1}{c}{ Subtests } & Media & DE & $\mathrm{Z}$ \\
\hline "Madre Teresa de Calcuta" & 1,02 &, 77 & $5,16^{* * *}$ \\
"Marie Curie" & 1,94 & 1,17 & $4,06^{* * *}$ \\
"Johannes Gutenberg" & 3,17 & 1,30 & $4,63^{* * *}$ \\
"Louis Pasteur" & 4,22 & 1,37 & $4,98^{* * *}$ \\
"Albert Einstein" & 6,88 & 2,32 & $2,65^{* * *}$ \\
Total & 17,25 & 5,31 & $1,79^{* *}$ \\
\hline
\end{tabular}

$* * \mathrm{p}<.01, * * * \mathrm{p}<.001, \mathrm{n}=613$ 


\section{Análisis inferencial}

Se comparó el nivel de comprensión lectora considerando el tipo de colegio (ver Tabla 4), encontrándose diferencias estadísticamente significativas entre los puntajes totales alcanzados por alumnos de cuarto año de secundaria de colegios estatales y no estatales $(\mathrm{Z}=-4,126 ; \mathrm{p}<, 001)$, observándose que quienes obtuvieron una media de rango más elevada fueron los alumnos de centros educativos no estatales. Estos resultados permiten señalar que los alumnos de cuarto año de secundaria de centros educativos no estatales presentan un mayor nivel de comprensión lectora que sus pares de centros educativos estatales.

Tabla N. ${ }^{\circ}$ 4. Comparación del nivel de comprensión lectora de los alumnos de cuarto año de secundaria considerando el tipo de colegio.

\begin{tabular}{|c|c|c|c|}
\hline $\begin{array}{c}\text { Estatal } \\
\mathrm{n}=397 \\
\text { Media de Rango }\end{array}$ & $\begin{array}{c}\text { No estatal } \\
n=216 \\
\text { Media de Rango }\end{array}$ & $\mathrm{U}$ & $\mathrm{Z}$ \\
\hline 285,26 & 346,96 & 34246 & $-4,126 * * *$ \\
\hline
\end{tabular}

Al compararse los puntajes alcanzados en cada uno de los subtests de la prueba considerando el tipo de colegio de procedencia (Tabla 5), se puede observar que se encontraron diferencias significativas en los subtests $1(Z=-3,958 ; p<, 001), 2(Z=-4,439 ; p$ $<, 001), 3(Z=-2,610 ; p<, 01)$ y $5(Z=-3,610 ; p<, 001)$, entre los alumnos de ambos tipos de colegio, siendo los estudiantes procedentes de colegios no estatales quienes obtienen medias de rango más altas, lo cual permite indicar que estos alumnos tienen un mayor nivel de comprensión lectora tanto a nivel literal como inferencial.

Tabla N. ${ }^{\circ}$ 5. Comparación del nivel de comprensión lectora de los alumnos del cuarto año de secundaria según el puntaje en cada uno de los subtests considerando la variable tipo de colegio.

\begin{tabular}{|c|c|c|c|c|}
\hline Subtests & Tipo de centro educativo & Media de rango & $\mathrm{U}$ & $\mathrm{Z}$ \\
\hline $\begin{array}{l}\text { Subtest } 1 \\
\text { "Madre Teresa de Calcuta" }\end{array}$ & $\begin{array}{c}\text { Estatal } \\
\text { No Estatal }\end{array}$ & $\begin{array}{l}287,39 \\
343,04\end{array}$ & 35091,000 & $-3,958 * * *$ \\
\hline $\begin{array}{l}\text { Subtest } 2 \\
\text { "Marie Curie" } \\
\end{array}$ & $\begin{array}{c}\text { Estatal } \\
\text { No Estatal } \\
\end{array}$ & $\begin{array}{l}284,26 \\
348,80 \\
\end{array}$ & 33847,000 & $-4,439 * * *$ \\
\hline $\begin{array}{l}\text { Subtest } 3 \\
\text { "Johannes Gutenberg" }\end{array}$ & $\begin{array}{c}\text { Estatal } \\
\text { No Estatal }\end{array}$ & $\begin{array}{l}293,56 \\
331,70 \\
\end{array}$ & 37540,000 & $-2,610 * *$ \\
\hline $\begin{array}{l}\text { Subtest } 4 \\
\text { "Louis Pasteur" }\end{array}$ & $\begin{array}{c}\text { Estatal } \\
\text { No Estatal }\end{array}$ & $\begin{array}{l}302,57 \\
315,14\end{array}$ & 41117,500 &,- 861 \\
\hline $\begin{array}{l}\text { Subtest } 5 \\
\text { "Albert Einstein" }\end{array}$ & $\begin{array}{c}\text { Estatal } \\
\text { No Estatal }\end{array}$ & $\begin{array}{l}288,11 \\
341,72\end{array}$ & 35375,500 & $-3,610 * * *$ \\
\hline
\end{tabular}

$* * \mathrm{p}<, 01, * * * \mathrm{p}<, 001$ 
En cuanto al análisis de diferencias en el desarrollo de la comprensión lectora de los alumnos varones y mujeres, los resultados encontrados (ver Tabla 6) indican que no existen diferencias significativas entre los alumnos de cuarto año de secundaria cuando se considera la variable sexo al comparar el puntaje total en la prueba.

Tabla N. ${ }^{\circ}$ 6. Comparación del nivel de comprensión lectora de los alumnos del cuarto año de secundaria considerando la variable sexo.

\begin{tabular}{cccc}
\hline $\begin{array}{c}\text { Varones } \\
\mathrm{n}=284 \\
\text { Media de Rango }\end{array}$ & $\begin{array}{c}\text { Mujeres } \\
\mathrm{n}=329 \\
\text { Media de Rango }\end{array}$ & $\mathrm{U}$ & $\mathrm{Z}$ \\
\hline 315,91 & 299,31 & 44187 & $-1,159$ \\
\hline * $<<, 05$ & & &
\end{tabular}

$\mathrm{Al}$ comparar los puntajes obtenidos en cada subtest entre los estudiantes varones y mujeres (ver Tabla 7), tampoco se encontraron diferencias estadísticamente significativas entre ambos grupos de participantes.

Tabla N. ${ }^{\circ}$ 7. Comparación del nivel de comprensión lectora de los alumnos del cuarto año de secundaria según el puntaje en cada uno de los subtests considerando la variable sexo.

\begin{tabular}{|c|c|c|c|c|}
\hline Subtests & Sexo & Media de rango & $\mathrm{U}$ & Z \\
\hline \multirow{2}{*}{$\begin{array}{l}\text { Subtest } 1 \\
\text { "Madre Teresa de Calcuta }\end{array}$} & Varones & 313,62 & \multirow{2}{*}{44838} & \multirow{2}{*}{,- 916} \\
\hline & Mujeres & 301,29 & & \\
\hline \multirow{2}{*}{$\begin{array}{l}\text { Subtest } 2 \\
\text { "Marie Curie" }\end{array}$} & Varones & 301,64 & \multirow{2}{*}{45196,5} & \multirow{2}{*}{,- 717} \\
\hline & Mujeres & 311,62 & & \\
\hline \multirow{2}{*}{$\begin{array}{l}\text { Subtest } 3 \\
\text { "Johannes Gutenberg" }\end{array}$} & Varones & 315,39 & \multirow{2}{*}{44334,5} & \multirow{2}{*}{$-1,117$} \\
\hline & Mujeres & 299,76 & & \\
\hline \multirow{2}{*}{$\begin{array}{l}\text { Subtest } 4 \\
\text { "Louis Pasteur" }\end{array}$} & Varones & 309,34 & \multirow{2}{*}{46054} & \multirow{2}{*}{,- 312} \\
\hline & Mujeres & 304,98 & & \\
\hline \multirow{2}{*}{$\begin{array}{l}\text { Subtest } 5 \\
\text { "Albert Einstein" }\end{array}$} & Varones & 320,04 & \multirow{2}{*}{43013,5} & \multirow{2}{*}{$-1,708$} \\
\hline & Mujeres & 295,74 & & \\
\hline
\end{tabular}

$* \mathrm{p}<, 05$

Al comparar los niveles de comprensión lectora considerando el tipo de colegio, se encontraron diferencias estadísticamente significativas entre los alumnos de colegios estatales y no estatales, siendo los alumnos de centros educativos no estatales quienes presentan un mayor nivel de comprensión lectora que sus pares de centros estatales. Por tal razón se han elaborado baremos diferenciados para los estudiantes de ambos tipos de colegios de Lima Metropolitana (Ver anexo 1). 


\section{DISCUSIÓN}

En cuanto los resultados del análisis de ítems de la Prueba de Comprensión Lectora DET para el cuarto año de secundaria, se encontraron valores significativos que permiten aceptar todos los ítems que conforman cada uno de los cinco subtests. Asimismo, el coeficiente alfa de Cronbach alcanzado en el análisis de la confiabilidad indica que el instrumento es confiable por el método de consistencia interna. En cuanto a la validez de constructo, se encontró que el modelo de un factor es válido, estos resultados permiten señalar que la prueba es válida, con lo cual cumple con los requerimientos psicométricos básicos para este tipo de instrumentos (Martínez Arias, 1985; Muñiz, 1996; Anastasi y Urbina, 1998).

Los resultados del análisis de la bondad de ajuste a la curva normal, realizados a través de la prueba de Kolmogorv-Smirnov mostraron que los valores de los estadísticos .S Z eran significativos, lo que permitió concluir que las distribuciones de los valores analizados no se aproximaban a la curva de distribución normal, por lo tanto se utilizaron estadísticos no paramétricos en el análisis de los datos (Siegel y Castellan, 1995).

Al comparar los niveles de comprensión lectora de los alumnos de cuarto año de secundaria considerando el tipo de colegio, se encontró que los alumnos de centros educativos no estatales presentan un mayor nivel de comprensión lectora, tanto a nivel literal como inferencial, lo cual indica que estos alumnos tiene una mayor capacidad que sus pares de colegios estatales para recordar las escenas presentadas en el texto y para repetir tanto las ideas principales como los detalles y las secuencias de los acontecimientos. Además, los participantes de centros no estatales mostraron mayor capacidad que los de centros estatales para captar las claves contextuales al interior del texto, para reconstruir el significado de la lectura, para relacionarlo con sus experiencias personales como con los conocimientos previos que poseen del tema, para plantearse hipótesis o inferencias que exigen la comprensión del texto de manera global y para reconocer las intenciones del autor como la superestructura del texto, es decir, tienen una mayor capacidad para aportar sus conocimientos al texto que leen y tomar una postura frente a lo expresado en él (Sánchez, 1998; Catalá, et al. 2001; Sánchez, 2004 y Amorós, 2006). Estos resultados ponen de manifiesto que el poder utilizar procesos y desarrollar estrategias para mejorar la comprensión lectora se encuentra relacionado con aquello que el maestro enseña en el colegio, porque es allí donde los estudiantes aprenden a aplicarlos para poder lograr el nivel de comprensión lectora que se espera tengan según el año de estudios en que se encuentran, esto coincide con lo que proponen autores como Echevarría y Gastón (2004), Vieiro y Gómez (2004), Vallés (2005) y Fons (2006), entre otros. Finalmente, es interesante señalar que no se observaron diferencias estadísticamente significativas en el nivel de comprensión lectora entre los alumnos y las alumas del cuarto año de secundaria.

\section{CONCLUSIONES}

1. La prueba de Comprensión Lectora DET para cuarto año de secundaria es un instrumento confiable.

2. La prueba de Comprensión Lectora DET para cuarto año de secundaria presenta validez de constructo. 
3. Se encontraron diferencias estadísticamente significativas en los niveles de comprensión lectora entre los alumnos de centros educativos no estatales y estatales.

4. Los estudiantes de cuarto año de secundaria de colegios no estatales presentan una mayor capacidad para recordar escenas, repetir las ideas principales, los detalles y las secuencias de los acontecimientos, que los participantes de colegios estatales.

5. Los alumnos de cuarto año de secundaria de centros educativos no estatales poseen mayor capacidad que los estudiantes de centros estatales para captar las claves contextuales al interior del texto, reconstruirlo y relacionarlo tanto con sus experiencias personales como con los conocimientos previos que poseen acerca del tema.

6. Los participantes de colegios no estatales tienen mayor capacidad para plantearse hipótesis o inferencias que implican la comprensión global del texto, que sus pares de colegios estatales.

7. Los estudiantes de centros educativos no estatales mostraron mayor capacidad que los de centros estatales para reconocer las intenciones del autor como la superestructura del texto.

8. Los alumnos de cuarto de secundaria de colegios estatales tienen mayor capacidad para tomar una postura frente a lo expresado en un texto.

9. No se encontraron diferencias estadísticamente significativas en el desarrollo de la comprensión lectora entre los alumnos varones y mujeres.

10. Se han elaborado baremos percentilares considerando el tipo de centro educativo.

\section{REFERENCIAS BIBLIOGRÁFICAS}

1. Alarcón, R. (2008). Métodos y diseños de investigación del comportamiento. Lima: Editorial Universitaria.

2. Almeyda, O. y Yataco, L. (1999). Las nuevas tecnologías y la comprensión lectora. Lima: F.A.M.

3. Amorós, M. (2006). Leer es comprender. En: Revista Signo Educativo, Mayo, XV (147), 1.22 .

4. Anastasi, A. y Urbina, S. (1998). Tests psicológicos. México: Editorial Prentice Hall.

5. Byrne B. M. (1989). A primer of LISREL: Basic applications and programming for Confirmatory Analytic Models. New York: Springer-Verlag Inc.

6. Byrne B. M. (1998). Structural equation modeling with LISREL, PRELIS, AND SIMPLIS: Basic concepts, applications and programming. New Jersey: Lawrence Erlbaum Associates Publishers.

7. Cairney, T. H. (1992). Enseñanza de la comprensión lectora. Madrid: Ediciones Morata S.A. 
COMPARACIÓN DE LA COMPRENSIÓN LECTORA EN ALUMNOS DE $4 .^{\circ}$ DE SECUNDARIA, DE CENTROS EDUCATIVOS ESTATALES Y NO ESTATALES

8. Catalá, G.; Catalá, M.; Molina, E. y Monclús, R. (2001). Evaluación de la comprensión lectora. Barcelona: Editorial GRAÓ.

9. Defior, S. (2000). Las dificultades de aprendizaje: Un enfoque cognitivo. Lectura, escritura, matemáticas. Granada: T.G. Arte, Juberías \& Cía, S.L.

10. Delgado, A., Escurra, L. y Torres, W. (2006). La Medición en Psicología y Educación: Teoría y aplicaciones. Lima: Editorial Hozlo S.R.L.

11. Dubois, E. (1991). El proceso de la lectura: De la teoría a la práctica. Buenos Aires: Editorial Aique.

12. Echevarría, M. y Gastón, I. (2004). Dificultades de la comprensión lectora en estudiantes. Escuela del Magisterio de Bízcala. Disponible en: www.vc.ehu.es/ campus/centro/e.g.b/

13. Fons, M. (2006). Leer y escribir para vivir. Alfabetización inicial y uso real de la lengua escrita en la escuela. Barcelona: Editorial GRAÓ.

14. Gandolfi, G. (2002). Comprensión lectora. La dimensión comunicativa de un aprendizaje cultural. Buenos Aires: Editorial Santillana.

15. Grimaldo, M. (2002). Adaptación del Cuestionario de Reflexión Socio Moral (SROM) de Gibas y Widaman. Universidad de San Martín de Porres. Lima: Rastros Gráficos S. A.

16. Kline, P. (1986). A handbook of test construction. Introduction to psychometric design. New York: Methuen \& Co. Ltd.

17. Kline, P. (1995). The handbook of psychological testing. London: Routledge.

18. Marín, G. (1986). Consideraciones metodológicas básicas para conducir investigaciones psicológicas en América Latina. En: Acta Psiquiátrica y Psicológica de América Latina, 32, 183-192.

19. Martínez Arias, R. (1985). Psicometría: Teoría de los tests psicológicos y educativos. Madrid: Síntesis.

20. Muñiz, J. (1996). Psicometría. Madrid: Editorial Universitas S.A.

21. Sánchez, L. (2004). El desafío de enseñar la comprensión lectora. Novedades Educativas, Mayo, 161, 6-11.

22. Sánchez, D. (1998). Cómo leer mejor. Lima: INLEC.

23. Sánchez, H. y Reyes, C. (2006). Metodología y diseños en la investigación científica. Lima: Editorial Visión Universitaria.

24. Siegel, S. y Castellan, N. (1995). Estadística no paramétrica: Aplicada a las ciencias de la conducta. México: Editorial Trillas.

25. Vallés, A. (2005). Comprensión lectora y procesos psicológicos. Liberabit, 11(11), 49-61.

26. Vieiro, P. y Gómez, I. (2004). Psicología de la lectura. Madrid: Pearson Prentice Hall. 